\title{
A novel approach to non-biased systematic random sampling: A stereologic estimate of Purkinje cells in the human cerebellum
}

\author{
Rajiv M. Agashiwalaa ${ }^{a}$ Elan D. Louis ${ }^{b}$, Patrick R. Hof ${ }^{c}$, and Daniel P. Perl ${ }^{a}{ }^{*}$ \\ aDepartment of Pathology, Neuropathology Division, Mount Sinai School of Medicine, One \\ Gustave L. Levy Place, Box 1134, New York, NY 10029-6574, USA \\ ${ }^{b}$ GH Sergievsky Center, Department of Neurology, Taub Institute for Research on Alzheimer's \\ Disease and the Aging Brain, College of Physicians and Surgeons, Columbia University, and \\ Department of Epidemiology, Mailman School of Public Health, Columbia University, 710 West \\ 168th Street, New York, NY 10032, USA
}

'Department of Neuroscience, Mount Sinai School of Medicine, New York, NY 10029, USA

\begin{abstract}
Non-biased systematic sampling using the principles of stereology provides accurate quantitative estimates of objects within neuroanatomic structures. However, the basic principles of stereology are not optimally suited for counting objects that selectively exist within a limited but complex and convoluted portion of the sample, such as occurs when counting cerebellar Purkinje cells. In an effort to quantify Purkinje cells in association with certain neurodegenerative disorders, we developed a new method for stereologic sampling of the cerebellar cortex, involving calculating the volume of the cerebellar tissues, identifying and isolating the Purkinje cell layer and using this information to extrapolate non-biased systematic sampling data to estimate the total number of Purkinje cells in the tissues. Using this approach, we counted Purkinje cells in the right cerebella of four human male control specimens, aged 41, 67, 70 and 84 years, and estimated the total Purkinje cell number for the four entire cerebella to be 27.03, 19.74, 20.44 and 22.03 million cells, respectively. The precision of the method is seen when comparing the density of the cells within the tissue: $266,274,173,166,167,603$ and $183,575 \mathrm{cells} / \mathrm{cm}^{3}$, respectively. Prior literature documents Purkinje cell counts ranging from 14.8 to 30.5 million cells. These data demonstrate the accuracy of our approach. Our novel approach, which offers an improvement over previous methodologies, is of value for quantitative work of this nature. This approach could be applied to morphometric studies of other similarly complex tissues as well.
\end{abstract}

\section{Keywords}

Stereology; Cerebellum; Brain; Methodology; Essential tremor

\section{Introduction}

In recent years it has been acknowledged that the use of one or two "representative sections" of a structure to provide quantitative morphometric assessments (e.g., the extent of lesion involvement, neuronal loss) may lead to serious inaccuracies in the results obtained (Perl et al., 2000). In the context of neurobiologic research, techniques that evaluate only

(C) 2008 Elsevier B.V. All rights reserved.

*Corresponding author. Fax: +1 212996 1343. daniel.perl@mssm.edu (D.P. Perl). 
"representative" sections are incapable of differentiating between a reduction in neuron number, shrinkage of neurons, and other differential changes between subregions of a structure, and thus do not truly and accurately represent the tissue. Furthermore, it is impossible to establish if the few sections being evaluated are truly representative of the entire structure being sampled.

These inaccuracies have been addressed through the use of non-biased systematic sampling and the techniques of stereology. The sampling techniques of stereology are intended to allow one to accurately determine the total number of objects present within a structure based on an approach employing non-biased systematic sampling (Gundersen et al., 1988a,b; Pakkenberg et al., 1991; Coggeshall and Lekan, 1996; West, 1999; Schmitz and Hof, 2000, 2005). This technique can only be performed on regions in which the entire structure is available for sampling. The overall principle of such sampling is that any object within a structure under examination will have an equal probability of being sampled using a probe that counts objects without bias according to an object's size. The principles of nonbiased systematic sampling, on which stereology is based, require that the boundaries of a structure being counted be rigorously definable. Accordingly, neuroanatomically distinct structures (e.g., hippocampus, entorhinal cortex, substantia nigra pars compacta) can be subjected to stereologic analysis, however structures without distinct boundaries present difficult problems that must be overcome to achieve accurate stereologic results.

Additionally, sampling problems arise in using these approaches within certain brain regions where the objects being counted are selectively arranged in a highly complex physical configuration within a very limited portion of the particular sample. A particular example of this phenomenon is seen in the Purkinje cells of the cerebellum, which only exist within a single, extremely complex, convoluted layer of the tissue, between the granular and molecular layers. Problems are also seen in the volumetric data, because in all previous studies (Nairn et al., 1989; Mayhew et al., 1990; Mayhew, 1991; West, 1991; Andersen et al., 1992, 2003), the volume of the sample was obtained by the Cavalieri method, which is based on the cross-sectional areas of serially produced blocks multiplied by the thickness of those blocks. This poses a significant problem if the tissue is complex and convoluted, as in the case of the cerebellum.

In the context of a project to quantify Purkinje cells in patients with essential tremor, a highly prevalent neurological disorder with emerging cerebellar pathology (Louis, et al., 2006a,b, 2007; Axelrad et al., 2008), we sought to obtain accurate stereologic estimates of Purkinje cells within the cerebellum. Purkinje cell stereologic estimates have never been performed on essential tremor cases in the past, and only a handful of other publications have performed estimates of Purkinje cells using stereologic probes (Nairn et al., 1989; Mayhew et al., 1990; Mayhew, 1991; West, 1991; Andersen et al., 1992, 2003). These studies concluded with a wide range of results and other accuracy issues. In order to address the above issues and obtain valid estimates of the total number of Purkinje cells in human brain specimens, we developed a new methodology. Here, we describe the approach we have taken and provide data on four control subjects.

\section{Results}

Four male control hemicerebella were evaluated, ages $41,67,70$ and 84 years at the time of death (Table 1). The samples of the hemicerebella weighed 53.8, 61.1, 57.2 and $62.9 \mathrm{~g}$, respectively. Assuming symmetry for the cerebellum, the weights of the whole cerebella would be 107.6, 127.2, 122.2 and $125.8 \mathrm{~g}$, respectively. The volumes of the hemicerebella were $50.8,57.0,61.0$, and $60.0 \mathrm{~cm}^{3}$, (estimated whole cerebellar volumes of 101.6, $114.0,122.0$ and $120.0 \mathrm{~cm}^{3}$ respectively). The hemicerebella were cut into eight blocks and 
the volume of each of the eight blocks added together to check for discrepancies. These summed volumes were 49.0, 52.9, 60.7 and $57.2 \mathrm{~cm}^{3}$, producing a difference of $1.8(3.5 \%)$, $4.1 \mathrm{~cm}^{3}(7.2 \%), 0.3(0.5 \%)$, and $2.8(4.7 \%) \mathrm{cm}^{3}$ respectively from the hemicerebellar volumes. The total Purkinje cell number in an entire cerebellum for the first specimen was 27.03 million cells, for the second specimen, 19.74 million cells, for the third specimen, 20.44 million cells, and for the fourth specimen, 22.03 million cells. The average Purkinje cell count per gram of cerebellum was $251,412,155,303,167,383$, and 175,223 respectively, and the average Purkinje cell count per $\mathrm{cm}^{3}$ of cerebellum was 266,274, 173,176, 167,603, and 183,575 , respectfully. The coefficient of variation (CV) between the Purkinje cell counts was 0.13 , with a mean of 22.31 million cells and a standard deviation of 2.85 million cells. The average coefficient of error (CE) across the four cases is $0.26 \%$, calculated via the Schmitz-Hof method (Schmitz and Hof, 2000). These data are shown in Table 2.

\section{Discussion}

While many methods of estimating Purkinje cell numbers in the cerebellum have been employed in the past, including the $\mathrm{Nv} \times \mathrm{Vref}$ method and the optical fractionator method, we have devised a novel approach to the calculation of the estimate, relying on volumetric measurements of the specimens. We first cut the hemicerebellum into eight blocks, and the volume of each of these blocks is measured by water displacement, and then systematically cut into $100 \mu \mathrm{m}$-thick sections, as described in Experimental procedures. A section is then randomly chosen from each of the eight blocks, the Purkinje cell layer is identified and traced using a computer software, and the optical fractionator is used to obtain an accurate estimate of the Purkinje cell number within that section. The volume of each counted section is calculated by multiplying its area as calculated by the computer software and its thickness. A new proportional variable called the Tissue Volume Coefficient is calculated by dividing the block's volume by the volume of the corresponding sectional volume (Average Block Volume/Section Volume=Tissue Volume Coefficient). Multiplying this coefficient by the Purkinje cell count obtained from each slide produces a total count of Purkinje cells for the corresponding block (Tissue Volume Coefficient Total Purkinje Cells in Section=Number of Purkinje Cells in Block). By following this procedure for all eight blocks, and the eight block estimates, an estimate of the total number of Purkinje cells within the entire cerebellar hemisphere is obtained.

Using this method of calculations, one can see how volumetric measurements are a critical component to extrapolate stereologically-based estimates to objects in three dimensions. The method traditionally employed for this volumetric calculation is the Cavalieri principle, which allows for accurate volume estimates, thus explaining its extensive use in previous studies. However, the application of the Cavalieri method to an extremely complex and convoluted structure, such as the cerebellum, proved impractical as the grid required to take into account the detailed architecture would be extremely fine, rendering quantification cumber-some and inefficient. Estimates may be obtained using a larger, and thus more manageable grid, but doing so would impact on the estimate obtained. Previous studies that necessitated calculating the volume of the cerebellum did so using the Cavalieri principle for their volume calculations. Alternatively, water displacement, due to the pervasive nature of water, provides an accurate and efficient method of measuring of the cerebellum's total volume.

The earliest stereologically-based estimate of Purkinje cells in the entire human cerebellum was performed by Nairn et al. (1989). These authors calculated a mean of 15.4 million cells in 12 human cerebella, with a range of 9.7 to 20.4 million cells. In their study, the mean fixed cerebellar weight was $119 \mathrm{~g}$, however there was a large range of values (88.8-148.8 
g), raising concerns that some specimens were not derived from control brains or that there was significant variability in either the dissection or preparation of the samples.

Subsequently, Andersen (1992), published a stereologically-based study of five human cerebella. This study determined the cerebellar volume using the Cavalieri method and they reported considerable variability with Purkinje cell number ranging from 24.6 to 34.2 million cells, with a mean of 30.5 million cells $(\mathrm{CV}=.13)$. Despite the similarity in our coefficients of variation, the authors raised concern about this variability and noted that their results should only be considered as "rough estimates" of the Purkinje cell population. A subsequent report by this group (Andersen et al., 2003) continued to employ the Cavalieri method to determine cerebellar volumes. They reported a mean total Purkinje cell count of 28 million $(\mathrm{CV}=0.16)$ in 19 human samples.

Our approach differs from these previous studies with regard to the determination of volume as well as isolation of the Purkinje cell layer as the counting volume in which sampling is performed. Since accurate volume measurements translate to accurate stereologic results, anything that can be done to better approximate the volume will result in more precise counts. The use of water displacement for the determination of volume provides a simple and accurate method independent of tissue complexity. Also, isolating the Purkinje cell layer from its surroundings allows for the optical fractionator probe to be confined to an area in which a true random sampling can occur, as opposed to the entire tissue, as was done in all previous papers, which took into account a disproportionate amount of area where there was no chance of finding a Purkinje cell, and thereby skewing the estimates. For example, Andersen et al. (2003) recognized limitations with stereologic counting of Purkinje cells across the entire cerebellum, stating that, "Many counting fields contained no Purkinje cells. Therefore, in order to sample at least 50-120 cells, 150-300 disectors had to be sampled...." This led to the use of excessively high numbers of counting frames in an attempt to offset the problem of empty frames. This creates an unnecessary burden and is a rather inefficient method of counting. Our approach of isolating the Purkinje cell layer affords us an increased level of efficiency because it avoids the problems of increasing counting frame numbers, the sampling area being much smaller and far better defined, thus addressing the problems encountered by Andersen et al.

Our Purkinje cell counts fall within the range of the previously estimated values for this cell population. Our counts also demonstrate high precision, with a low coefficient of error (C. E.). Thus, this novel approach can be used particularly for quantitative work involving complex samples, but also for morphometric studies of all types, such as on tissue samples from various anatomical origins, including but not limited to, estimates of objects in specific anatomical structures within the cerebellum or any other structurally complex organ. A confounding variable in this data is age, which seems to have an impact on the Purkinje cell number, independent of the mass or volume of the cerebellum. As there may be a relationship between age and Purkinje cell number, additional studies on a larger cohort which includes a broader age range, are warranted.

In conclusion, the approach described here minimizes variability related to analysis of the Purkinje cells, which make up an extremely small proportion of the cerebellum. Our approach also addresses the concerns raised by previous researchers as to the multiple factors that impede accurate cell counts in such a convoluted, precise and complex tissue. These problems have hampered research into the potential role of Purkinje cell pathology in a number of important conditions, such as essential tremor and autism. Whereas this method certainly provides increased efficiency, determining an increase in the accuracy of the estimates compared to previous studies would require a substantially larger sample to be studied. This new method will now be used to study additional control and pathologic 
specimens in preparation for addressing the question of whether Purkinje cells are diminished in number in association with essential tremor and other neurodegenerative disorders.

\section{Experimental procedures}

Each sample was an intact, formalin-fixed, human cerebellar hemisphere, obtained from the Mount Sinai Medical Center/Bronx Veteran Affairs Medical Center Brain Bank. These samples were obtained from brains of deceased individuals who were without clinical or neuropathologic evidence of significant nervous system disease. Each sample was removed from the brainstem by cuts through the cerebellar peduncles and then cut mid-sagittally through the cerebellar vermis by the same person in an effort to standardize the sectioning, minimizing any inaccuracy in the sectioning and sample preparation process itself. The resulting hemicerebellum sample was then weighed, and the weight of the entire cerebellum was calculated by doubling that of the hemicerebellum. The volume of the hemicerebellum was then measured using water displacement (Archimedes' principle) (Scherle, 1970). This was achieved using a $1000 \mathrm{ml}$ graduated cylinder that contained $500 \mathrm{ml}$ of water. The cylinder was tipped to allow a gradual introduction of the cerebellar hemisphere into the water without any spillage. The new water level was read on the cylinder's graduations and, by subtracting $500 \mathrm{ml}$, the volume was ascertained. The volume measurement was taken twice and the two values were averaged. The cerebellar hemisphere was then cut in random coronal, parasagittal and horizontal planes with a Mopec AH028 Brain Knife, to yield 8 tissue blocks (Fig. 1). To ensure consistency in the volume measurement (i.e., that no significant amount of tissue had been lost during the sectioning process), the volume of each tissue block was measured in the same way. The agreement between the initial volumetric and this second (summed) measurement was high based on the Pearson's correlation coefficient $(r=0.95)$; we used the initial volume of the whole cerebellum in our calculations.

Each block was then cryoprotected by emersion in increasing aqueous concentrations of sucrose. Upon equilibrating in 30\% sucrose, each block was serially cryosectioned at 100 $\mu \mathrm{m}$ intervals using a Leica CM1900 motorized cryostat. Within each of the eight serial section sets, a random section was chosen for cell counting using a random number generator, producing eight sections, each section representative of its entire block of tissue. The selected sections were Nissl-stained (cresyl violet) and mounted on glass slides. Cell counting of the stained slides was performed on an Olympus BX51 Research Microscope fitted with an Optiscan II XYZ Motorized Stage Encoder System (Prior Scientific Inc, Rockland, MD) that was coupled with a computer running MicroBrightField (MBF Bioscience, Williston, VT) StereoInvestigator 6 software and a Wacom Interactive Pen Display, Model Cintiq 15UX.

The counting procedure began by drawing contour lines with the stereologic software in order to isolate the Purkinje cell layer from the surrounding cerebellar tissue. Contour lines within the StereoInvestigator software defined the counting area for the program so it could randomly select locations from within them to count, and as well use the selected area in the extrapolation of the counting data to render an accurate count (Glaser and Glaser, 2000) (Fig. 2). Once the Purkinje cell layer was isolated, the program's optical fractionator probe was selectively applied within these isolated contours. The optical fractionator probe randomly selected locations within each contour to place a 3 -dimensional $(300 \times 300 \times 30 \mu \mathrm{m})$ counting box, also known as an optical disector, within the defined tissue (Fig. 3). Any Purkinje cells within the dissector were counted using standardized stereologic criteria for inclusion-exclusion, where the nucleoli of cells that were either partially or completely within the counting frame were counted so long as they did not intersect the two exclusion lines that made up the lower and left borders of the counting frame (Sterio, 1984). The 
disector was placed regularly along the tissue at $x, y$ positions based on the grid that was placed on the tissue by the software. The initial point on the grid was randomly placed, and each $x, y$ position was systematically random from that point. From the cell counts within a contour, the software extrapolated an estimate of the number of Purkinje cells using the following formula:

$$
N=\sum Q^{-} \frac{t}{h} \cdot \frac{1}{a s f} \cdot \frac{1}{s s f}
$$

where $N$ is the estimated number of cells within the contour, $\Sigma Q^{-}$is the number of cells actually counted within the contour, $t$ is the original thickness of the tissue before processing, $h$ is the height of the disector, as $f$ is the area sampling fraction or the ratio between the area of the disector and the area of the sampling grid, that is, the size of the steps taken along the $x$ and $y$ axes: (a(disector) /a(x,y step $)$ ) and ssf is the section sampling fraction, which represents a fraction of the total number of disectors counted (West, 1991). This final variable, for our method, remained 1 , since each disector at each $x, y$ position was counted, and thus there was no interval between section sampling and the $s s f=1$. By adding the extrapolated Purkinje cell counts within each contour, a total number of Purkinje cells present within the entire section was calculated.

Subsequently, the area of the entire section was obtained by drawing a contour around its perimeter, allowing the software to calculate the area of that contour, thus enabling the total volume of the section to be calculated (the measured area in $\mu \mathrm{m}^{2}$ multiplied by the section thickness). Using the initially obtained volume measurement of the block and the nowobtained volume of the section from within that block, a new proportional variable called the Tissue Volume Coefficient was calculated by dividing the block's volume by the volume of the corresponding sectional volume (Average Block Volume/Section Volume=Tissue Volume Coefficient). Multiplying this coefficient by the Purkinje cell count obtained from each slide produced a total count of Purkinje cells for the corresponding block (Tissue Volume Coefficient-Total Purkinje Cells in Section=Number of Purkinje Cells in Block). By following this procedure for all eight blocks, and then combining all of the cell counts from all eight blocks, a total estimate of Purkinje cells within the entire cerebellar hemisphere was obtained. This number was then multiplied by two to yield the estimated Purkinje cell number for the entire cerebellum. Once the Purkinje cell estimates were calculated, they were divided by the volume of the entire cerebellum as well as the mass of the cerebellum to calculate densities of the Purkinje cells by volume and mass in order to explore numerical correlations between the densities of different cerebella. The coefficient of error (CE) was estimated for each case using the Schmitz-Hof method (Schmitz and Hof, 2000). The CE for each contour was determined and then the mean was calculated giving the $\mathrm{CE}$ for the case. The coefficient of variation (CV) was also calculated across cases.

\section{Acknowledgments}

We gratefully acknowledge the excellent assistance of Dr. Dushyant P. Purohit, Ms. Amy Hsu and Ms. Sandy Harry in carrying out this work. We thank Ms. Vina Ayers for her assistance with Fig. 1. This study was supported by funds from NIH grants NS042859 (Dr. Louis), AD02219, AG05138 and MH058911 (Drs. Perl, Hof).

\section{REFERENCES}

Andersen BB, Korbo L, Pakkenberg B. A quantitative study of the human cerebellum with unbiased stereological techniques. J. Comp. Neurol 1992;326:549-560. [PubMed: 1484123]

Andersen BB, Gundersen HJ, Pakkenberg B. Aging of the human cerebellum: a stereological study. J. Comp. Neurol 2003;466:356-365. [PubMed: 14556293] 
Axelrad JE, Louis ED, Honig LS, Flores I, Ross GW, Pahwa R, Lyons KE, Faust PL, Vonsattel JPG. Reduced Purkinje cell number in essential tremor. Arch. Neurol 2008;65:101-107. [PubMed: 18195146]

Coggeshall RE, Lekan HA. Methods for determining numbers of cells and synapses: a case for more uniform standards of review. J. Comp. Neurol 1996;364:6-15. [PubMed: 8789272]

Glaser GR, Glaser GM. Stereology, morphometry, and mapping: the whole is greater than the sum of its parts. J. Chem. Neuroanat 2000;20:115-126. [PubMed: 11074348]

Gundersen HJ, Bagger P, Bendtsen TF, Evans SM, Korbo L, Marcussen N, Moller A, Nielsen K, Pakkenberg B. The new stereology tools: disector, fractionator, nucleator and point sampling intercepts and their use in pathological research and diagnosis. Acta Pathol. Microbiol. Immunol. Scand 1988a;96:857-881.

Gundersen HJ, Bendtsen TF, Korbo L, Marcussen N, Moller A, Nielsen K, Nyengaard JR, Pakkenberg B. Some new, simple and efficient stereology methods and their use in pathological research and diagnosis. Acta Pathol. Microbiol. Immunol. Scand 1988b;96:379-394.

Louis ED, Vonsattel JPG, Honig LS, Lawton A, Moskowitz C, Ford B, Frucht S. Essential tremor associated with pathological changes in the cerebellum. Arch. Neurol 2006a;63:1189-1193. [PubMed: 16908751]

Louis ED, Vonsattel JPG, Honig LS, Ross GW, Lyons KE, Pahwa R. Neuropathological findings in essential tremor. Neurology 2006b;66:1756-1759. [PubMed: 16769958]

Louis ED, Faust PL, Vonsattel JPG, Honig LS, Rajput A, Robinson CA, Rajput A, Pahwa R, Lyons KE, Ross GW, Borden S, Moskowitz CB, Lawton A, Hernandez N. Neuropathological changes in essential tremor: 33 cases compared with 21 controls. Brain 2007;130:3297-3307. [PubMed: 18025031]

Mayhew TM. Accurate prediction of Purkinje cell number from cerebellar weight can be achieved with the fractionator. J. Comp. Neurol 1991;308:162-168. [PubMed: 1890236]

Mayhew TM, MacLaren R, Henery CC. Fractionator studies on Purkinje cells in the human cerebellum: numbers in right and left halves of male and female brains. J. Anat 1990;169:63-70. [PubMed: 2200769]

Nairn JG, Bedi KS, Mayhew TM, Campbell LF. On the number of Purkinje cells in the human cerebellum: unbiased estimates obtained by using the "fractionator". J. Comp. Neurol 1989;290:527-532. [PubMed: 2613942]

Pakkenberg B, Moller A, Gundersen HJG, Mouritzen Dam A, Pakkenberg H. The absolute number of nerve cells in substantia nigra in normal subjects and in patients with Parkinson's disease estimated with an unbiased stereological method. J. Neurol. Neurosurg. Psychiatry 1991;54:30-33. [PubMed: 2010756]

Perl DP, Good PF, Bussière T, Morrison JH, Erwin JM, Hof PR. Practical approaches to stereology in the setting of aging- and disease-related brain banks. J. Chem. Neuroanat 2000;20:7-19. [PubMed: 11074340]

Schmitz C, Hof PR. Recommendations for straightforward and rigorous methods of counting neurons based on a computer simulation approach. J. Chem. Neuroanat 2000;20:93-114. [PubMed: 11074347]

Schmitz C, Hof PR. Design-based stereology in neuroscience. Neuroscience 2005;130:813-831. [PubMed: 15652981]

Scherle W. A simple method for volumetry of organs in quantitative stereology. Mikroskopie 1970;26:57-60. [PubMed: 5530651]

Sterio DC. The unbiased estimation of number and sizes of arbitrary particles using the disector. J. Microsc 1984;134:127-136. [PubMed: 6737468]

West MJ. Unbiased stereological estimation of the total number of neurons in the subdivisions of the rat hippocampus using the optical fractionator. Anat. Rec 1991;231:482-497. [PubMed: 1793176]

West MJ. Stereological methods for estimating the total number of neurons and synapses: issues of precision and bias. Trends Neurosci 1999;22:51-61. [PubMed: 10092043] 


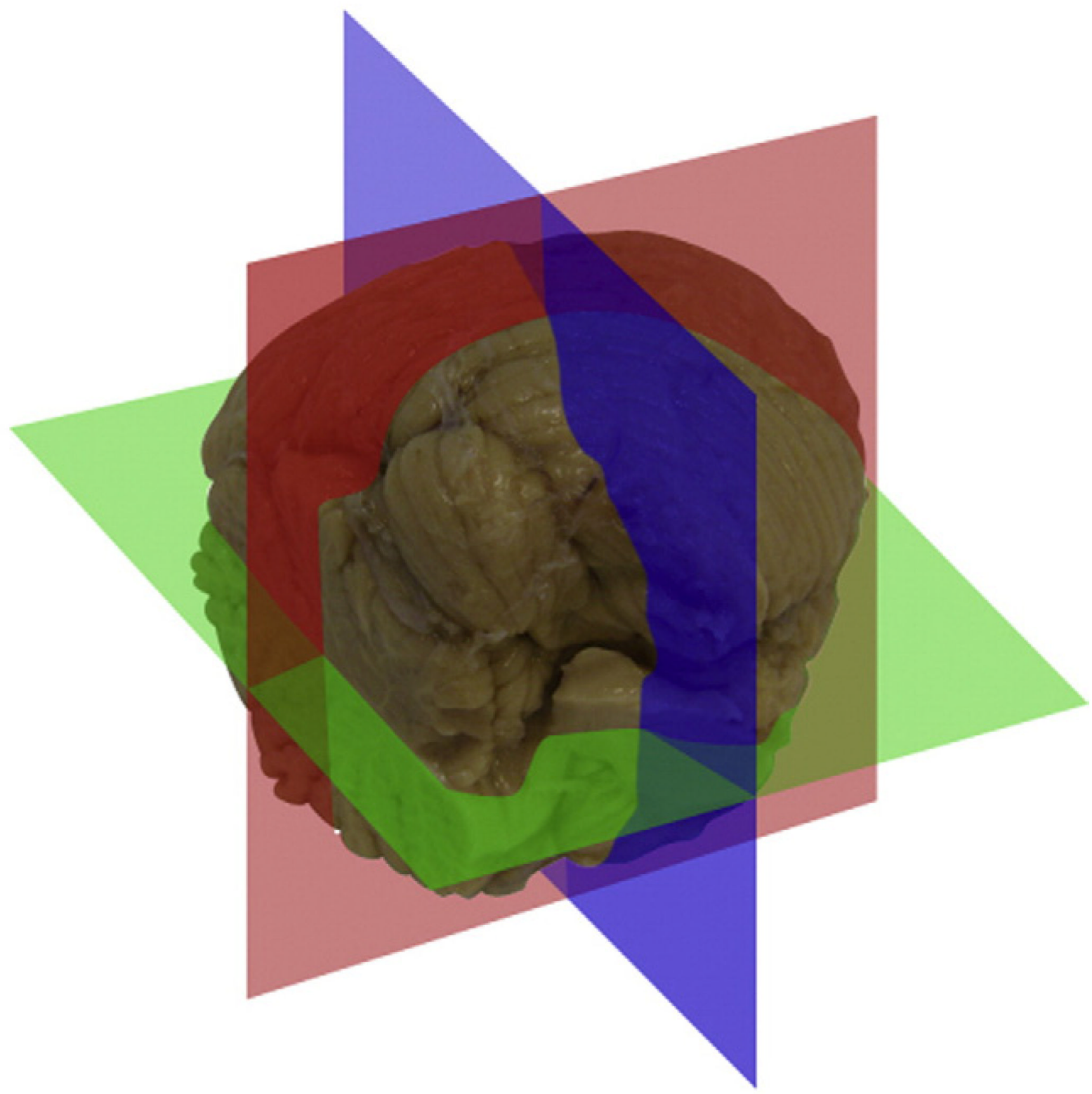

Fig. 1.

The hemicerebellum is subdissected into 8 blocks using random cuts along the coronal, sagittal and horizontal planes. 

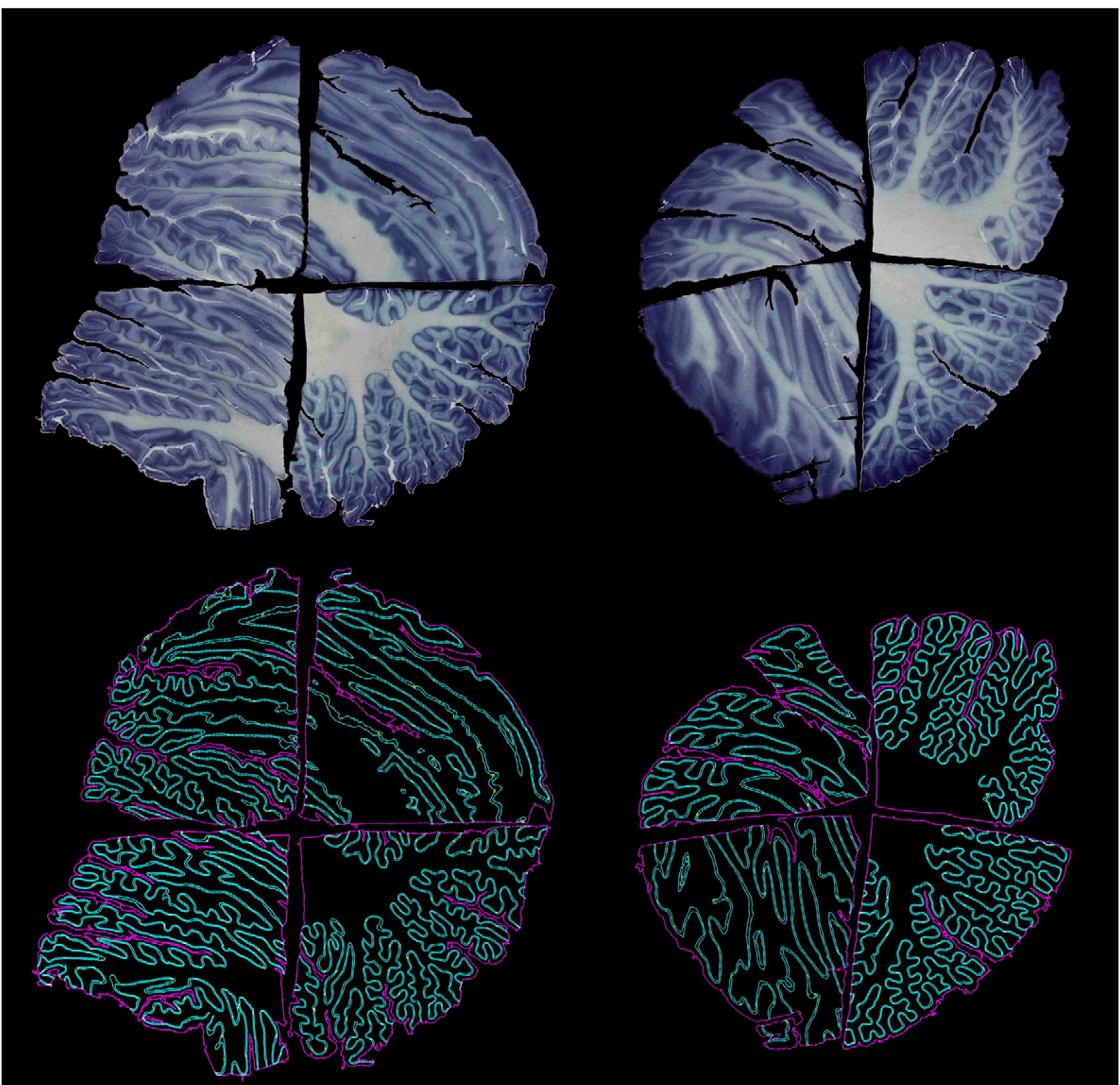

Fig. 2.

Eight sections showing histologic and contour views. Notice the isolation of the Purkinje cell layer in between the molecular and internal granular layers. 


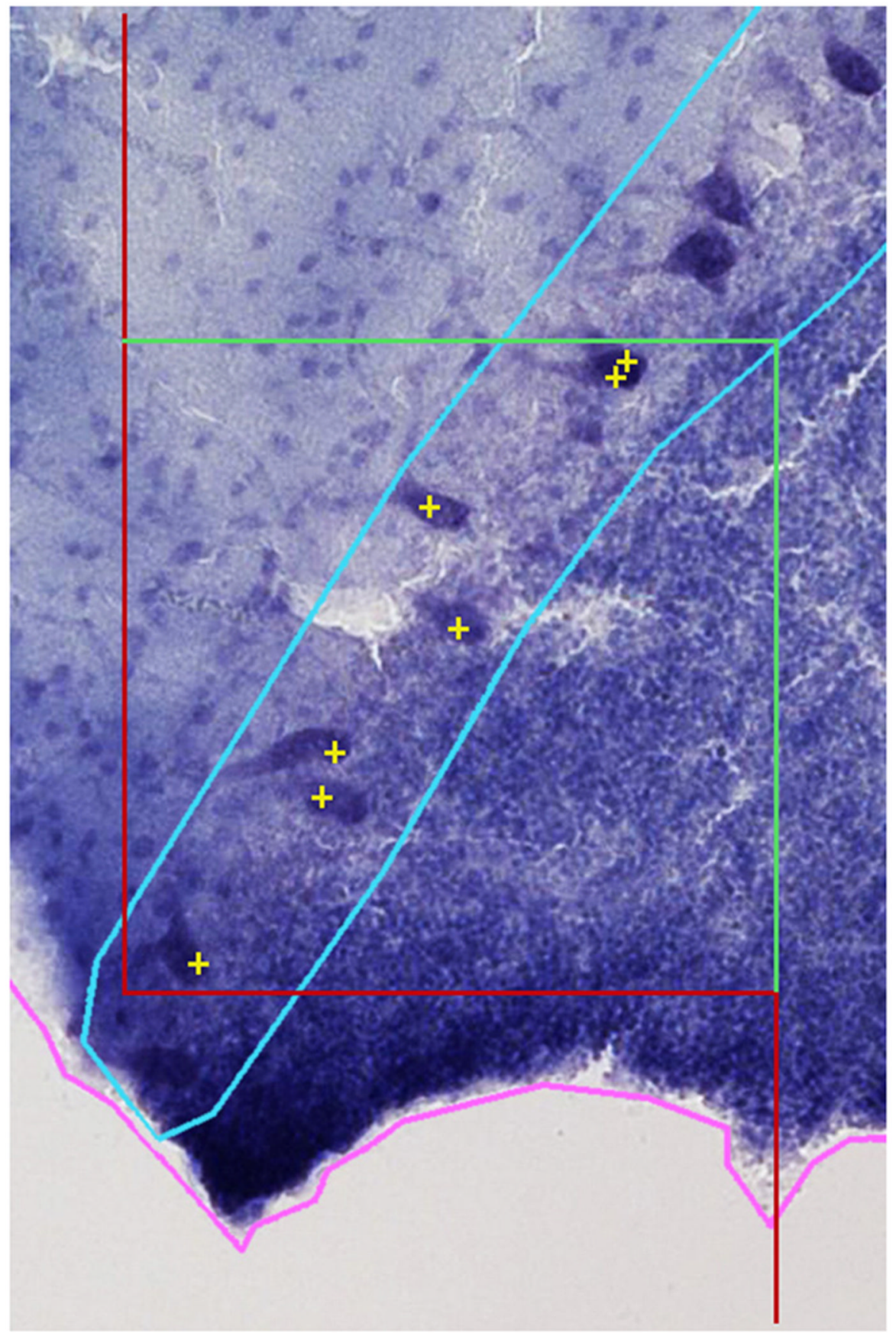

Fig. 3.

Stereologic disector containing Purkinje cells marked for counting. 
Table 1

Case Summary

\begin{tabular}{lcclc}
\hline $\begin{array}{l}\text { Subject } \\
\text { no. }\end{array}$ & Gender & $\begin{array}{c}\text { Age } \\
\text { years) }\end{array}$ & Cause of death & $\begin{array}{c}\text { Postmortem } \\
\text { interval (hours) }\end{array}$ \\
\hline 1 & M & 41 & $\begin{array}{l}\text { Suicide by } \\
\text { hanging } \\
\text { Cardiopulmonary } \\
\text { arrest }\end{array}$ & 10.6 \\
2 & M & 67 & 9.3 \\
3 & M & 70 & $\begin{array}{l}\text { Cardiopulmonary } \\
\text { arrest }\end{array}$ & 17.3 \\
4 & M & 84 & $\begin{array}{l}\text { Cardiopulmonary } \\
\text { arrest }\end{array}$ & 8.5 \\
\hline
\end{tabular}

Brain Res. Author manuscript; available in PMC 2010 August 25. 
